\title{
Avaliação da utilização da pós cura nas propriedades térmicas e mecânicas de um sistema epóxi de cura à temperatura ambiente
}

\section{Evaluation of the post cure use on the thermal and mechanical properties of a room temperature curing epoxy system}

Gabriel Benedet Dutra ${ }^{1}$, Robson Tureck Zargiski ${ }^{2}$, Thiago Antonio Fiorentin ${ }^{1}$, Robin Bahr Junor ${ }^{2}$, Pedro Ferreira Zanneti ${ }^{2}$

\footnotetext{
${ }^{1}$ Centro Tecnológico de Joinville, UFSC, Rua Dona Francisca, 8300 - Bloco U, Joinville, Santa Catarina, Brasil. e-mail: gabriel.d@ufsc.br

${ }^{2}$ Euro \& Zanetti Análises Térmicas, Rua São Paulo, Bairro Jardim Guanabara, Goiânia, Goiás, Brasil. e-mail: robin.bahr@euroezanetti.com.br
}

\section{RESUMO}

Neste trabalho, uma resina epóxi de cura a temperatura ambiente foi submetida a ciclos térmicos de pós cura para verificar as alterações nas propriedades térmicas e mecânicas finais do material. As amostras foram obtidas por vazamento em molde metálico e os ciclos de pós cura foram realizados a $75^{\circ} \mathrm{C}, 90^{\circ} \mathrm{C}, 110^{\circ} \mathrm{C}$ por uma hora e um ciclo de $110^{\circ} \mathrm{C}$ por 2 horas. Os resultados de calorimetria exploratória diferencial (DSC) mostraram que a temperatura de transição vítrea do material $(\mathrm{Tg} \infty)$ não sofreu alteração significativa com a realização destes ciclos de pós cura, indicando um número de ligações cruzadas muito similar a amostra sem pós cura. Os resultados de termogravimetria (TGA) revelaram uma degradação térmica em etapas; onde cerca de $70 \%$ em massa do material sofre degradação térmica na faixa de temperaturas de $330,9^{\circ} \mathrm{C}$ até $345,1^{\circ} \mathrm{C}$. Foi utilizado o diagrama tempo temperatura transformação (TTT) de um polímero termofixo para entendimento da cinética de cura, onde foi sugerido que a quantidade de resina e agente endurecedor teve uma influência mais significativa na cura plena da amostra do que os ciclos de pós cura adotados neste trabalho. Em termos de propriedades mecânicas, a dureza do material permaneceu inalterada com a realização dos ciclos de pós cura. Somente um grupo de amostra obteve menores valores de dureza pois o agente de cura em excesso funcionou com agente plastificante. Em termos de módulo de elasticidade, o método de vigas livres apresentou valores entre $1,72 \mathrm{GPa}$ e $2,18 \mathrm{GPa}$, e os ciclos de pós cura não tiverem nenhuma influência notável nesta propriedade.

Palavras-chave: Pós-cura, resina epóxi, propriedades térmicas, propriedades mecânicas.

\section{ABSTRACT}

In this work, a room temperature curing epoxy system was submitted to post cure cycles to evaluate the thermal and mechanical properties of the material. The samples were obtained by casting into a metallic tool and the post cure cycles were performed at $75^{\circ} \mathrm{C}, 90^{\circ} \mathrm{C}, 110^{\circ} \mathrm{C}$ for one hour and at $110^{\circ} \mathrm{C}$ cycle for 2 hours. The differential scanning calorimetry (DSC) results showed that the glass transition temperature of the material have not change significantly with the post cure cycles, which indicates a number of molecular crosslinks very similar to the original sample, without post cure. The thermogravimetric (TGA) results revealed a stepwise thermal degradation; where about $70 \%$ mass of the material undergoes thermal degradation in the temperature range from $330.9^{\circ} \mathrm{C}$ to $345.1^{\circ} \mathrm{C}$. The temperature-time transformation (TTT) diagram of a thermoset polymer was used to understand the cure kinetics, where it was suggested that the amount of resin and curing agent had a more significant influence on the full curing of the sample than the post cure cycles carried out in this article. By means of mechanical properties, the hardness of the material remained unchanged with the post curing cycles. Only one batch obtained lower values of hardness because the excess of curing agent 
worked as plasticizing agent. O In terms of elastic modulus, the free-beam method presented values between $1.72 \mathrm{GPa}$ and $2.18 \mathrm{GPa}$, and the post cure cycles did not have any notable influence on this property.

Keywords: Post-cure, epoxy resin, thermal properties, mechanical properties.

\section{INTRODUÇÃO}

Em vários segmentos da indústria a utilização de polímeros termorrígidos a base de resinas epóxi vem sofrendo um significativo aumento nas últimas décadas, desde recobrimentos anticorrosivos [1], adesivos [2], materiais eletrônicos [3], aeronáuticos / aeroespaciais [4], área de transporte entre outros [5]. As principais razões para este crescimento provem da funcionalidade do anel epóxi neste polímero, o qual pode abrir e reagir com vários agentes de cura, resultando em diferentes propriedades físicas, mecânicas e térmicas do material [6]. Para alcançar as propriedades finais, um polímero termorrígido deve passa por uma reação química de polimerização ou cura. A cura pode ser dividida nas etapas de: reação química (liquido viscoso), seguido de gelificação e posteriormente a vitrificação (sólido vítreo) [7]. Durante a cura a resina passa por uma contração dimensional devido à aproximação das moléculas principais do polímero e tensões residuais podem estar presentes na matriz.

Além disto, algumas resinas durante as etapas de cura podem apresentar alta exotermia de reação, emissão de voláteis e consequente formação de bolhas. A cinética da reação de cura e os parâmetros utilizados são fatores determinantes e podem interferir de forma significativa nas propriedades finais da resina. Em muitos casos, a cura pode acontecer de forma incompleta, isto acontece devido à elevada viscosidade durante etapa de gelificação, o que dificulta a movimentação molecular e a formação de ligações cruzadas. Nestes casos uma reticulação adicional é necessária para completar a formação da rede. Para isto é realizado um aquecimento posterior de pós cura de forma a permitir que o sistema obtenha um maior grau de reticulação e consequente melhor desempenho térmico e estrutural [8].

Ademais, as resinas epóxis ainda podem ser divididas em sistemas que curam a temperatura ambiente e sistemas que exigem calor para curar. A maioria dos sistemas de dois componentes são formulados para curar a temperatura ambiente e somente requerem a mistura da resina com o agente de cura. A vantagem principal destes sistemas é a forma de processamento que requer menor gasto energético. Por outro lado, para aplicações de alto desempenho, a realização de um aquecimento posterior, como a pós cura, em sistemas que curam a temperatura ambiente pode modificar as propriedades da resina. Entretanto, a literatura e a indústria ainda carecem de estudos sistemáticos acerca deste assunto [5]. Assim, este artigo apresenta uma investigação da influência de diferentes ciclos de pós cura, em termos de temperatura e tempo, em uma resina epóxi comercial desenvolvida para curar a temperatura ambiente. As caracterizações realizadas visam identificar o efeito do aquecimento em termos de propriedades térmicas e mecânicas finais da resina. As amostras curadas a temperatura ambiente e pós curadas foram avaliadas em termos de temperatura de transição vítrea $(\mathrm{Tg} \infty)$, através de análises de calorimetria exploratória diferencial (DSC) com duas rampas de aquecimentos para cada ciclo. Além disto, foram realizadas análises de termogravimetria (TGA) para identificação da temperatura de degradação e dureza Shore e ensaio não destrutível de viga livre para determinação do módulo de elasticidade.

\section{MATERIAIS E MÉTODOS}

As amostras foram confeccionadas com resina epóxi DGEBA 2004 transparente e agente de cura 3154, ambos da marca Redelease. De acordo com o fornecedor da resina, a $\mathrm{Tg} \infty$ da resina curada é em torno de $70^{\circ} \mathrm{C}$. A resina e o agente de cura foram misturados seguindo a proporção de $50 \mathrm{~g}$ de agente de cura para $100 \mathrm{~g}$ de resina, como sugerido pelo fabricante, através de agitação manual por 60 segundos. A mistura foi então vazada em um molde metálico. Sobre o molde foi previamente utilizada um agente desmoldante a base de cera de carnaúba da marca TecGlaze-N. Logo após o vazamento, uma bolsa de vácuo foi colocada sobre o molde e que foi selada fita Tacky-Tape. Durante a etapa de cura, foi estabelecida pressão absoluta de $630 \mathrm{mmHg}$ por 50 minutos com a utilização de uma bomba de vácuo. Logo após, as amostras foram deixadas dentro do molde por 24 horas a temperatura ambiente para finalização da etapa de cura.

A etapa de pós cura foi realizada com 4 (quatro) grupos de amostras, sendo que cada grupo apresenta 5 (cinco) corpos de prova. Com base na $\mathrm{Tg} \infty$ da amostra curada foram definidas as condições dos ciclos de pós-cura, assim as quais são apresentadas na Tabela 1. A rampa de aquecimento e a rampa de resfriamento teve uma taxa de aproximadamente $4 \% \mathrm{~min}$. A pós cura foi realizada em uma estufa com controle de temperatura e com atmosfera oxidativa.

As curvas de DSC foram obtidas com a utilização de um DSC 214 - Forno de prata fabricante 
NETZSCH. Os ciclos de térmico foi de $20{ }^{\circ} \mathrm{C}$ a $200{ }^{\circ} \mathrm{C}$, a uma taxa de aquecimento $20^{\circ} \mathrm{C} / \mathrm{min}$, seguido de resfriamento para $20{ }^{\circ} \mathrm{C}$ a uma taxa de $30^{\circ} \mathrm{C} / \mathrm{min}$, então segundo aquecimento a $20^{\circ} \mathrm{C} / \mathrm{min}$ até $200^{\circ} \mathrm{C}$ sem controle de resfriamento. A atmosfera utilizada foi de nitrogênio. Para determinação de temperaturas e entalpias relacionadas a eventos foi utilizada a norma ASTM E793-06 [9].

As curvas de TGA foram obtidas com um STA 409 - Forno de prata fabricante NETZSCH. As amostras foram aquecidas a uma taxa de $20^{\circ} \mathrm{C} / \mathrm{min}$ até $450^{\circ} \mathrm{C}$ no ar sintético.

Tabela 1: Identificação das amostras submetidas a diferentes ciclos de pós cura.

\begin{tabular}{l|l|l}
\hline \multirow{2}{*}{$\begin{array}{c}\text { IDENTIFICAÇÃO GRU- } \\
\text { PO }\end{array}$} & \multicolumn{2}{c}{ PÓS CURA } \\
\cline { 2 - 3 } & TEMPERATURA $\left({ }^{\circ} \mathbf{C}\right)$ & TEMPO (HORAS) \\
\hline SP & \multicolumn{2}{c}{ Sem pós cura } \\
\hline P75 & 75 & 1 \\
\hline P90 & 90 & 1 \\
\hline P110 & 110 & 1 \\
\hline P110-2 & 110 & 2 \\
\hline
\end{tabular}

A dureza Shore D foi medida em durômetro marca UHAG. Foram retiradas quinze medidas de diferentes corpos de prova de cada grupo de amostras e feito a média dessas medidas como resultado do ensaio de dureza. Os resultados serão apresentados em forma de histogramas e as barras no gráfico representam um intervalo de confiança para média ( $t$ student $95 \%$ ).

O módulo de elasticidade foi medido através do método da viga livre, o qual baseia-se na norma ASTM E 1876-01 [10]. O módulo de elasticidade é determinado utilizando a frequência de ressonância do modo de flexão da viga. Este método consiste em obter a frequência de ressonância de uma amostra através da excitação por um martelo de impacto. Utiliza-se um acelerômetro ou um microfone para captar a vibração, transformando-a em um sinal elétrico que será passado ao analisador de sinais [11]. Os resultados serão apresentados em forma de histogramas com a média de cinco amostras por condição e as barras no gráfico representam um intervalo de confiança para média ( $t$ student $95 \%$ ).

Os equipamentos utilizados no experimento são: um martelo de impacto; um microfone; um analisador de sinais; um suporte rígido que possa representar a amostra como uma viga livre-livre e um corpo de prova com formato de viga. A Figura 1 apresenta um esquema dos equipamentos utilizados e como eles são arranjados.

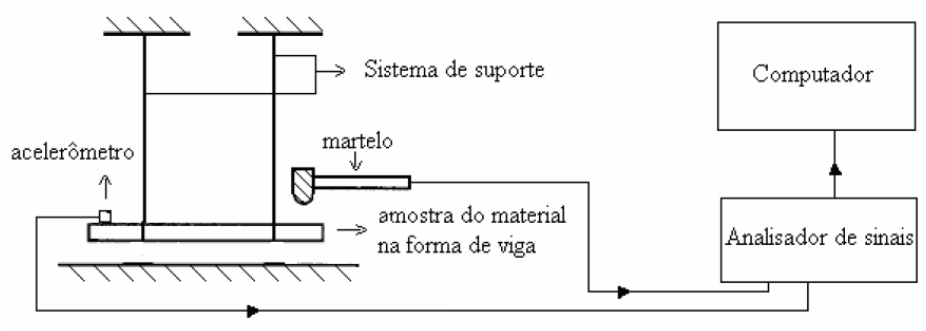

Figura 1: Esquema de ensaio de vibração método viga livre para obtenção do módulo de elasticidade [11].

Com as frequências de ressonância de flexão e torção, calcula-se os módulos de elasticidade (E). As equações para o cálculo dos módulos são fornecidas pela norma. Para o cálculo do módulo de elasticidade (E), tem-se:

$$
E=0,9465\left(\frac{m f^{2}}{b}\right)\left(\frac{L^{3}}{t k^{3}}\right) T 1
$$

Onde $T 1$ é um fator de correção para o modo de flexão que está relacionado com o coeficiente de Poisson, definido pela norma, e (b) e (t) são a largura e espessura da viga, respectivamente. A norma cita que se a ra- 
zão do comprimento pela espessura do corpo de prova (L/t) for maior ou igual a 20, o que realmente aconteceu nos corpos de prova ensaiados, $T 1$ pode ser calculado pela seguinte expressão:

$$
T 1=\left[1,000+6,585\left(\frac{t k}{L}\right)^{2}\right]
$$

\section{RESULTADOS E DISCUSSÕES}

Os resultados e discussões serão divididos de acordo com as análises térmicas para cinética de cura, onde serão abordados os experimentos de DSC e TGA e as análises mecânicas que trata da dureza e módulo de elasticidade.

\subsection{Análises térmicas}

Neste capítulo estão apresentados os resultados e discussões das técnicas de análises, em relação a temperatura de transição vítrea $(\operatorname{Tg} \infty)$ e calor residual da reação obtidas via DSC. Ademais, estão apresentados os resultados de TGA e uma discussão acerca do diagrama tempo temperatura e transformação (TTT) para um polímero termofixo.

As Figuras 2 e 3 mostram os resultados de DSC relativos ao aquecimento 1 e aquecimento 2 respectivamente. Nestes gráficos é possível identificar o ponto médio da $\mathrm{Tg} \infty$ e o calor residual de reação, representados de maneira comparativa na Figura 4.

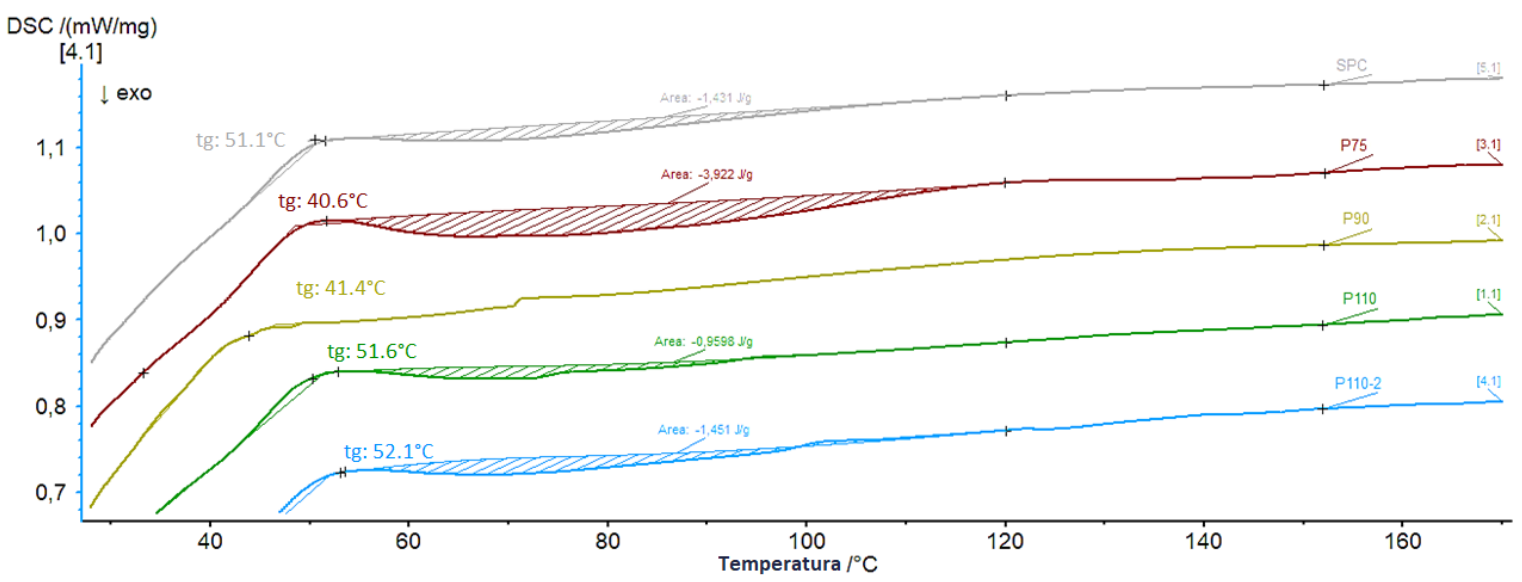

Figura 2: DSC referente ao ciclo térmico de aquecimento 1 das amostras.

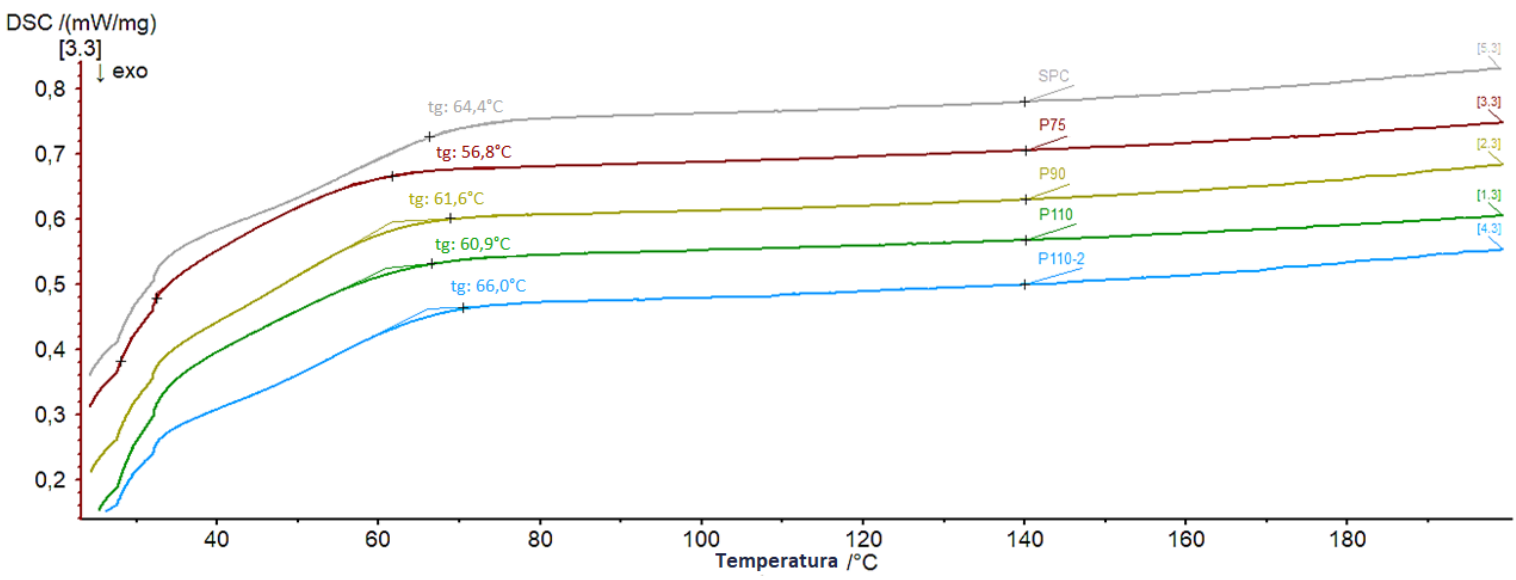

Figura 3: DSC referente ao ciclo térmico de aquecimento 2 das amostras. 
Também na Figura 4 estão os valores de $\mathrm{Tg} \infty$ estipulada no aquecimento 1 e do aquecimento 2. As barras apresentadas nas colunas representam +4 e $-4{ }^{\circ} \mathrm{C}$, conforme incerteza da técnica de medição da $\mathrm{Tg} \infty$. Ao analisar estes valores de temperatura das amostras sob diferentes condições de pós cura no aquecimento 1 , nenhuma mudança evidente foi constatada, todas as amostras apresentaram uma $\mathrm{Tg} \infty$ na faixa de $40{ }^{\circ} \mathrm{C}$ a $52{ }^{\circ} \mathrm{C}$ aproximadamente. A principal alteração da temperatura de $\mathrm{Tg} \infty$ foi observada ao comparar as amostras submetidas ao aquecimento 1 e ao aquecimento 2. Em todos os casos houve um significativo aumento no valor da temperatura. $\mathrm{O}$ ciclo 1 apresentou um aquecimento de até $200{ }^{\circ} \mathrm{C}$ nas amostras, seguido de resfriamento e posterior aquecimento 2 . Assim, todas as amostras do aquecimento 2 foram submetidas a um ciclo de pós cura de até $200{ }^{\circ} \mathrm{C}$, referente ao aquecimento 1 , alterando o valor da $\mathrm{Tg} \infty$ no material para uma faixa de $57{ }^{\circ} \mathrm{C}$ a $66{ }^{\circ} \mathrm{C}$ aproximadamente. Este aumento é de cerca de $35 \%$ em relação ao valor de Tg $\infty$ registrado no primeiro aquecimento para todas as amostras.

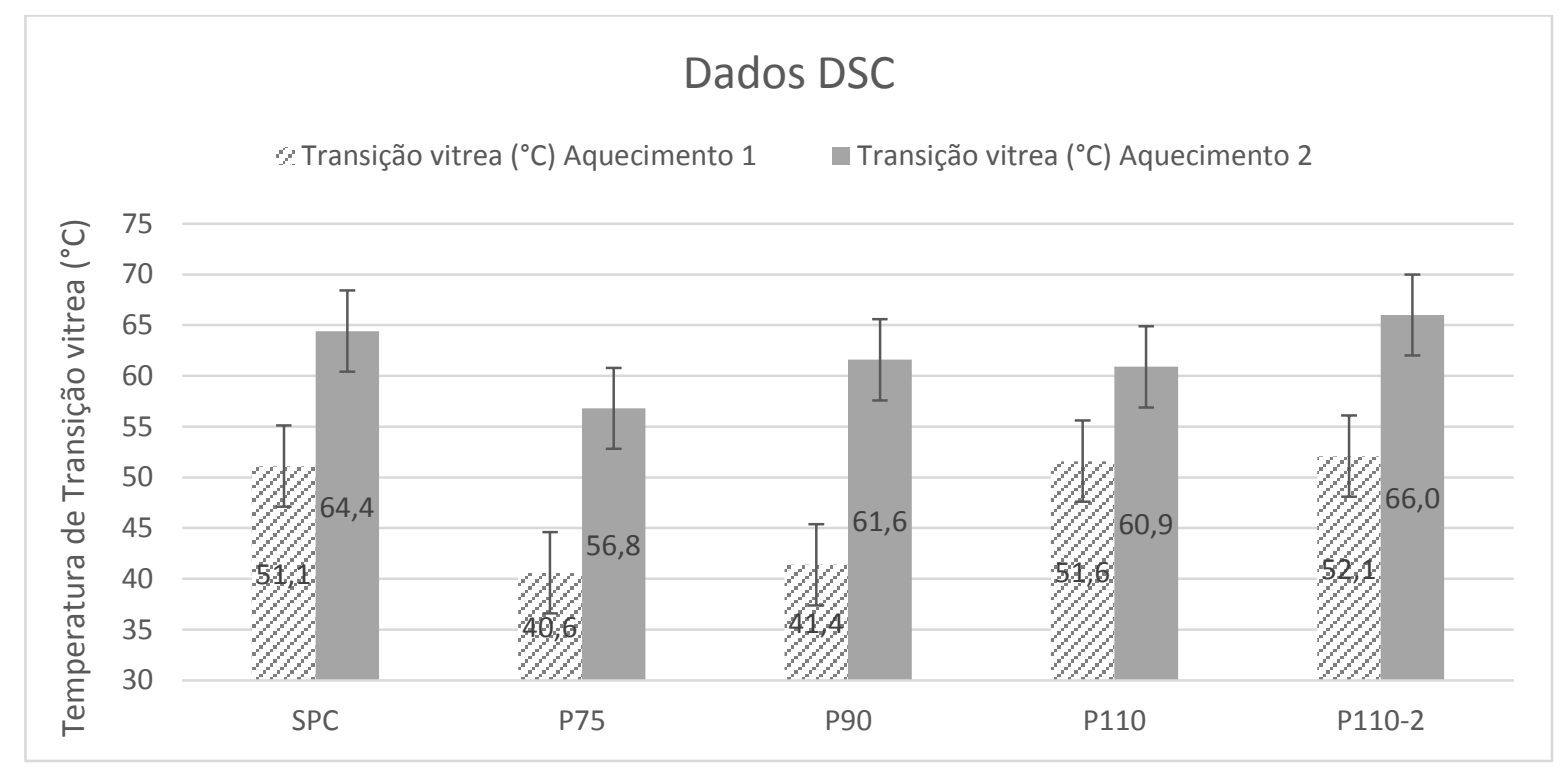

Figura 4: Valores de temperatura de transição vítrea provenientes das curvas de DSC.

Portanto, sugere-se que os valores escolhidos para janela experimental de pós cura deste trabalho, entre $75^{\circ} \mathrm{C}$ e $110^{\circ} \mathrm{C}$, não demonstraram significativa diferença em relação a quantidade de ligações cruzadas que afetassem a $\mathrm{Tg} \infty$ das amostras. Ao trabalhar com temperaturas mais elevadas estas mudanças seriam mais sensíveis na resina, porém vale ressaltar que além do aumento da $\mathrm{Tg} \infty$, altas temperaturas podem provocar uma degradação inicial do material e também a formação de ligações cruzadas oxidativas. CARBAS et al. [13] em seus estudos com adesivos a base de epóxi constataram que a em algumas faixas de temperatura de pós cura a $\operatorname{Tg} \infty$ do material se manteve inalterada e constante, podendo até sofrer um certo decréscimo devido a uma degradação.

A Tabela 2 mostra os dados de exotermia de residual de reação obtidos pelas curvas de DSC. Este valor refere-se a área integrada no gráfico do DSC no primeiro aquecimento, o que é representada em Joule por grama do material. Esta energia demonstra uma reação exotérmica, possivelmente proveniente de uma cura incompleta no material [8]. Nota-se que este vale é inexistente no aquecimento 2, sugerindo uma cura completa de todas as amostras após o aquecimento 1. As amostras SPC e P75 apresentaram os maiores valores de exotermia residual, 1,431 J/g e 3,922 J/g respectivamente. A única condição que não apresentou exotermia de cura no primeiro aquecimento foi a P90. Não houve uma relação direta ou tendência entre a temperatura de pós cura com a exotermia de reação. Outros fatores, como obtenção das amostras, heterogeneidade do sistema, entre outros, podem ter tido um efeito mais significativo. 
Tabela 2: Exotermia residual provenientes dos resultados de DSC.

\begin{tabular}{l|l|l}
\hline IDENTIFICAÇÃO & $\begin{array}{l}\text { EXOTERMIA RESIDUAL (J/GRAMA) } \\
\text { AQUECIMENTO 1 }\end{array}$ & $\begin{array}{l}\text { EXOTERMIA RESIDUAL (J/GRAMA) } \\
\text { AQUECIMENTO 2 }\end{array}$ \\
\hline SPC & 2,925 & 0,000 \\
\hline P75 & 3,922 & 0,000 \\
\hline P90 & 0,000 & 0,000 \\
\hline P110 & 0,960 & 0,000 \\
\hline P110-2 & 1,451 & 0,000 \\
\hline
\end{tabular}

A Figura 5 mostra os resultados da TGA, onde é ilustrado o gráfico de perda de massa em percentual das amostras em função da temperatura. Nesta mesma figura as perdas em massa foram divididas e estão indicadas para cada faixa de temperatura.

A primeira perda de massa acontece entre $40{ }^{\circ} \mathrm{C}$ e $150{ }^{\circ} \mathrm{C}$, respectiva a possível absorção de água ou emissão de voláteis. A segunda perda de massa acontece próximo a $300{ }^{\circ} \mathrm{C}$, que está atrelada a degradação de algum aditivo ou fração de menor peso molecular da resina. As temperaturas respectivas a terceira e maior perda de massa, assim como o percentual de massa residual das amostras após os ensaios estão separadamente demonstradas na Figura 6.

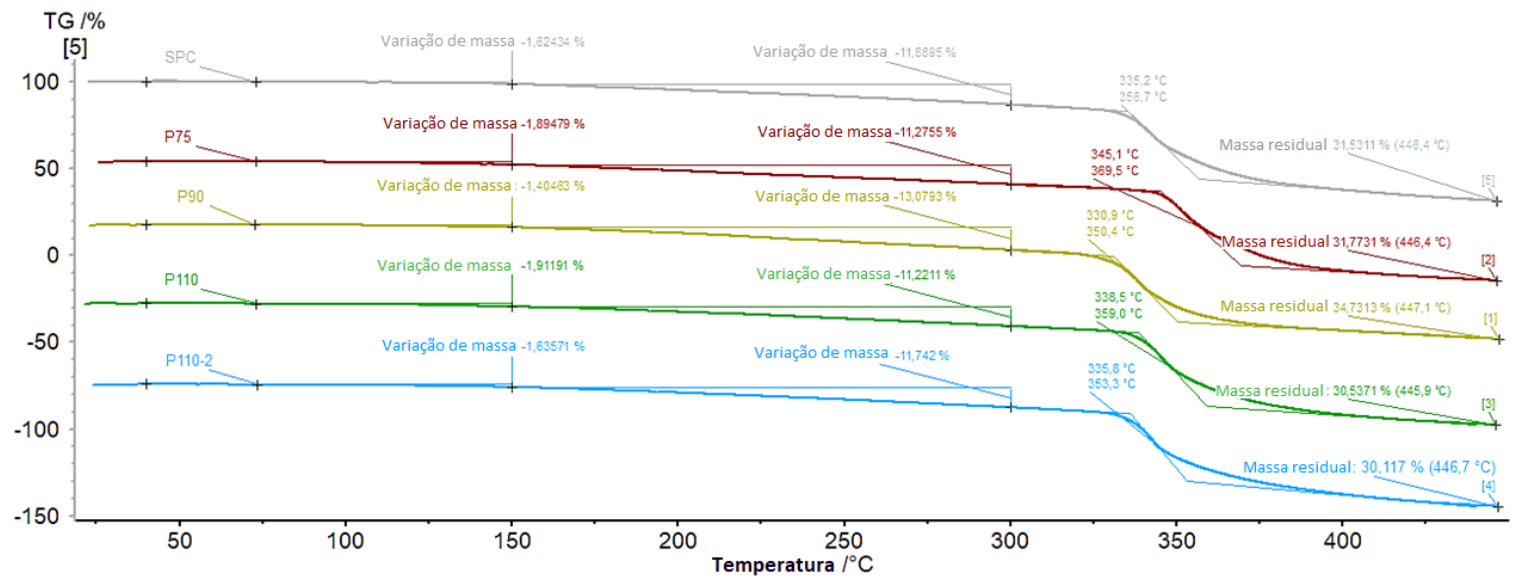

Figura 5: TGA referente ao ciclo térmico de aquecimento das amostras e perda de massa.

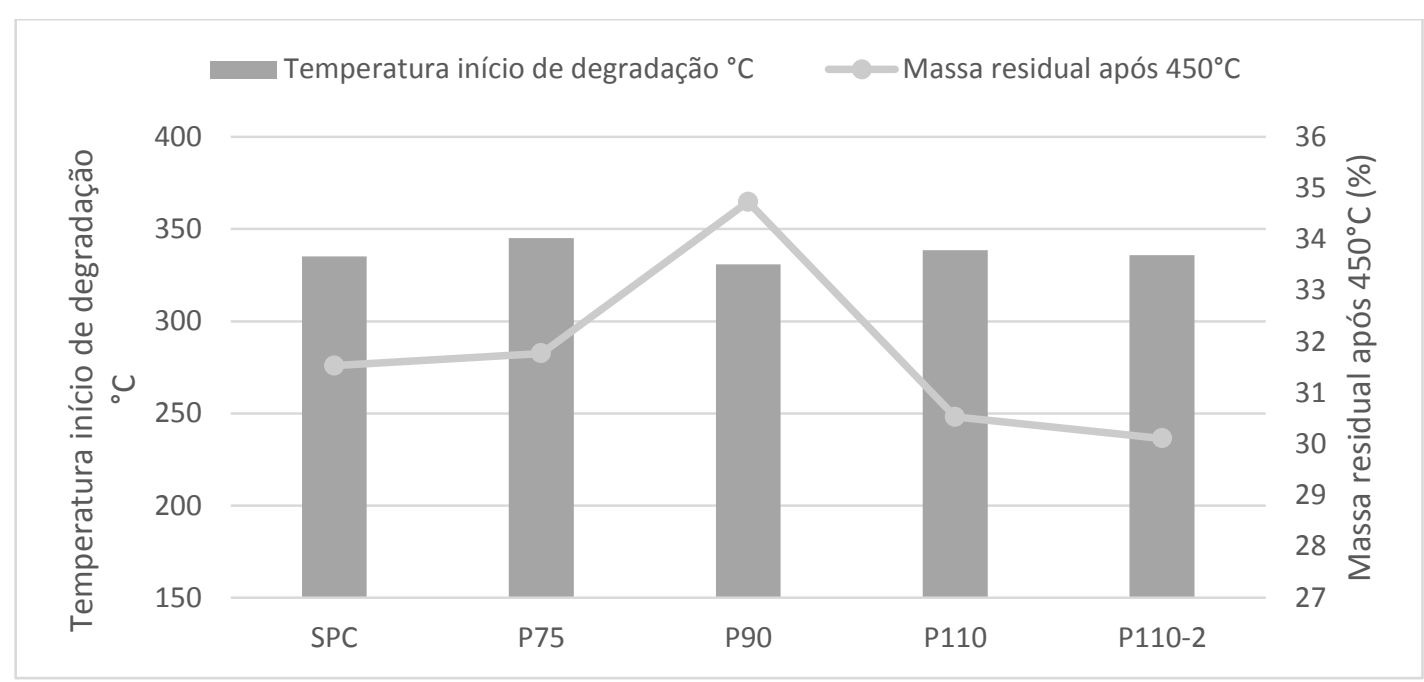

Figura 6: Temperatura de degradação e percentual de massa residual das amostras submetidas a diferentes ciclos de cura. 
Pelo gráfico de barras na Figura 6 não é possível constatar nenhuma influência evidente da realização da pós cura na temperatura de degradação da resina. Independente do tratamento realizado o rompimento das cadeias moleculares provocadas pelo calor ficou na faixa de $330,9{ }^{\circ} \mathrm{C}$ até $345,1{ }^{\circ} \mathrm{C}$. No mesmo gráfico é apresentada a massa residual das amostras após a pós cura. Ao preparar as amostras foi realizado uma mistura proporcional de $100 \mathrm{~g}$ de resina com $50 \mathrm{~g}$ de agente de cura, em percentual em massa representada por de $66,7 \%$ de resina e 33,3\% de agente de cura. A quantidade de massa residual mostrou um percentual em torno de 30-34\%. De acordo com o fornecedor, nesta resina não há presença de materiais inorgânicos.

Uma hipótese é que a resina começou o processo de degradação separadamente do agente de cura, sendo que a temperatura máxima escolhida pela análise não alcançou a temperatura de degradação do mesmo. Seguindo esta hipótese, todas as condições apresentaram uma quantidade de agente de cura, entre 30 e $32 \%$, exceto a amostra P90 que apresentou uma quantidade de 34,7\%. Assim, sugere-se que a formulação P90 apresenta a maior quantidade de agente de cura que as demais, o que pode estar atrelado ao processo produtivo da amostra. Esta quantidade de agente de cura pode também ter ocasionado uma cura mais plena da resina, pois ao observar o DSC da amostra P90, esta condição foi a única que não apresentou vale respectivo a exotermia de reação no aquecimento 1, vide Figura 2 e Tabela 2. Como comentado anteriormente, a existência deste vale estaria associada a uma cura não completa da resina. Entretanto, uma quantidade sobressalente de agente de cura pode proporcionar mudança nas propriedades físico mecânicos, o que será explorado no capítulo posterior.

Uma das formas de analisar os acontecimentos cinéticos que representam a cura é com a ajuda de diagramas de transformação isotérmicos de tempo e temperatura (TTT). Nestes diagramas estão demonstradas transformações de geleificação, vitrificação, cura completa e degradação da resina, em termos de temperatura e tempo. A curva de vitrificação em forma de $\mathrm{S}$ e a curva de geleificação dividem o gráfico TTT em quatro estados distintos relacionados ao processo de cura termofixo: região líquida, região borracha gel, região vítrea gel e região vítrea. A Figura 7 ilustra um diagrama TTT generalizado de uma resina. Além disto, o diagrama apresenta três temperaturas importantes, as quais são: $\operatorname{Tg} 0$ é a temperatura onde não existe qualquer tipo de reação, $\mathrm{Tg} \infty$ a temperatura de transição vítrea da resina totalmente curada, e gelTg é a temperatura onde a geleificação e vitrificação ocorrem simultaneamente, assim como o ponto onde as curvas de vitrificação e geleificação se cruzam [12].

Nos estágios iniciais da cura, antes da geleificação ou vitrificação, as reações são controladas pela cinética de química dos reagentes. Na região entre geleificação e vitrificação (região borrachosa) a reação ainda é controlada quimicamente, mas pode mudar de produto químico para controle por difusão, ou movimentação molecular. Quando a vitrificação ocorre, a reação torna-se a controlada por difusão, e a taxa de reação diminui em duas ou três ordens de grandeza abaixo daquelas nas regiões líquida ou gel, de modo que, para fins práticos a reação é essencialmente extinta logo após a vitrificação [8]. Devido a este fato, qualquer variável quantitativa de resina e agente de cura pode proporcionar modificações na resina mais expressivas que aquelas provocadas por uma pós cura de uma resina vitrificada. Assim sendo, sugere-se que as etapas iniciais da cinética de cura causaram uma influência mais evidente em termos de cura plena que o próprio ciclo térmico de pós cura, já que após a vitrificação a cinética de cura tem uma redução de até algumas ordens de grandeza em relação a etapa de reação química [12]. Desta forma, o cuidado para determinar as quantidades apropriadas de resina e agente de cura, assim como a homogeneização adequada, pode trazer mais benefícios para as propriedades térmicas do material que ciclos de pós cura em certas situações.

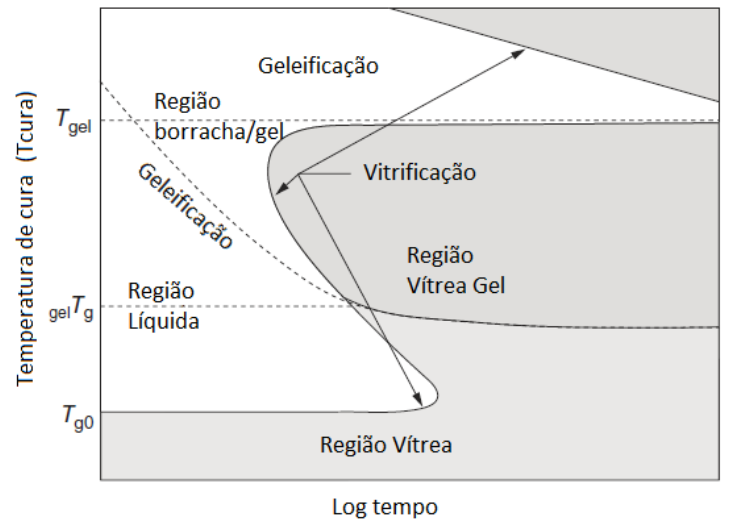

Figura 7: Diagrama tempo temperatura transformação (TTT) de um polímero termofixo, adaptado de Menczel (8). 


\subsection{Análises mecânicas}

As análises mecânicas foram realizadas através de ensaio de dureza Shore D e cálculo de módulo de elasticidade (E) via ensaio de rigidez vibração.

A Figura 8 apresenta os valores de dureza Shore D das amostras submetidas a diferentes ciclos de pós cura e a referência. Os valores variaram em uma faixa de 50 a 70 Shore D, dependendo da condição. O intervalo de confiança adotado ( $t$ student $95 \%$ ) mostrou que todas as condições apresentaram valores na mesma faixa de dureza, exceto as amostras do grupo P90.

A pós cura pode aumentar a mobilidade molecular de forma a aumentar a densidade de ligações cruzadas e também proporcionar um alivio de tensões na matriz polimérica. Como comentado anteriormente em relação as curvas de DSC, a Tg $\infty$ do material praticamente manteve-se inalterada, o que sugere uma quantidade de ligações cruzadas também muito similar entre todas as condições mesmo após os ciclos de pós cura. $\mathrm{Na}$ literatura, ZHANG et al. [14] desenvolveram um modelo de elementos finitos para investigar o nível de cura alcançado com diferentes ciclos térmicos da resina epóxi e também mediram a dureza Shore D. Os resultados variaram em uma faixa de 74 a 90 Shore $\mathrm{D}$, e como aqui reportados não tiveram uma influência direta da temperatura adotada. Um segundo efeito que também não foi observado foi o alivio de tensões provenientes da etapa de cura. Durante a cura, a resina forma ligações cruzadas entre as moléculas principais, o que proporciona uma contração tridimensional da amostra. Significativas tensões internas são desenvolvidas acima e abaixo da $\operatorname{Tg} \infty$, quando a resina é volumetricamente restrita [15]. Ao reaquecer o material até temperaturas próximas a $\mathrm{Tg} \infty$, a mobilidade atômica favorece uma acomodação das cadeias, o que reduz as tensões internas e pode diminuir a dureza da resina. Na literatura, SHOKRIEH et al. [16] reportaram em seu estudo uma redução das tensões residuais utilizando ciclos de pós cura em compósitos a base de resina epóxi.

Neste trabalho, nenhuma tendência clara de aumento ou redução de dureza foi observado com a realização dos ciclos de pós cura. A única constatação foi que houve uma queda de dureza no grupo de amostras P90. Esta diminuição de dureza deve estar atrelada a uma quantidade superior de agente de cura proveniente da etapa de obtenção dos corpos de prova, o que foi sugerido via análises de TGA. O excesso de agente de cura pode funcionar como agente plastificante, o que proporciona uma maior mobilidade das cadeias e reduz a dureza do material. AMARAL et al. [17] realizaram ensaios de tração em amostras de compósitos a base de resina epóxi e identificaram que o grupo de amostras que apresentou um excesso de agente de cura teve os menores valores de tensão máxima e tensão de escoamento, e a maior ductilidade. Estas propriedades foram atribuídas à presença de um excesso do agente de cura nas amostras atuando neste caso como plastificante da rede de ligações cruzadas.

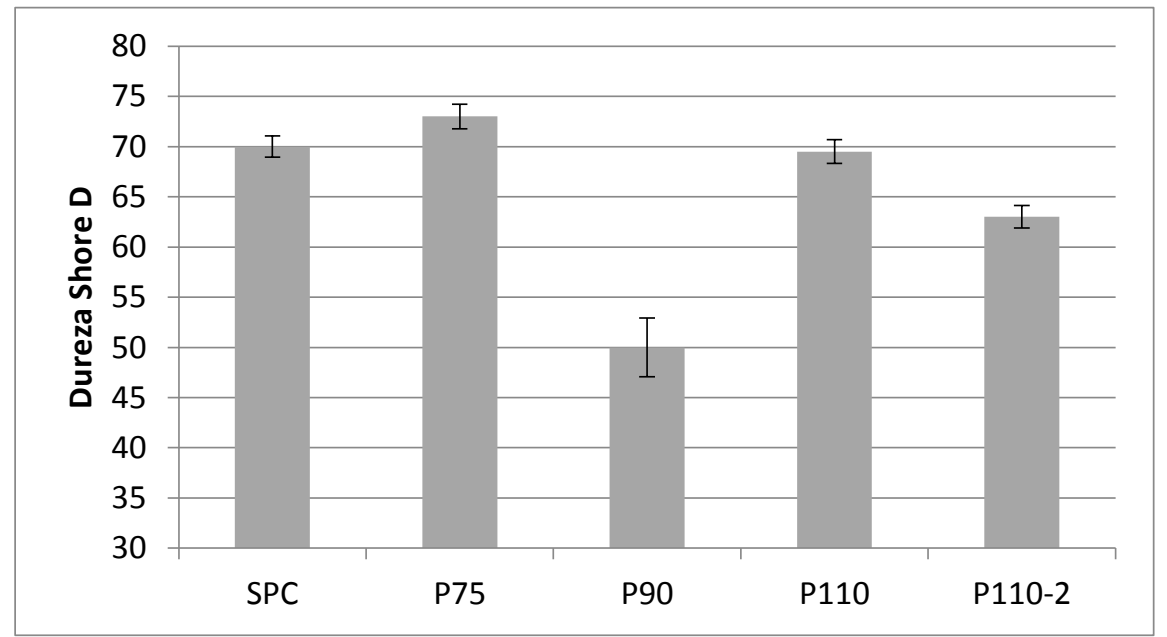

Figura 8: Dureza Shore D das amostras submetidas a diferentes ciclos de pós cura.

Outra propriedade mecânica avaliada foi o módulo de elasticidade (E) da resina após o processamento. Para verificação deste método foi utilizada a técnica de ensaio não destrutível via vibração. A Figura 8 apresenta os valores calculados de acordo com as equações (1) e (2). 


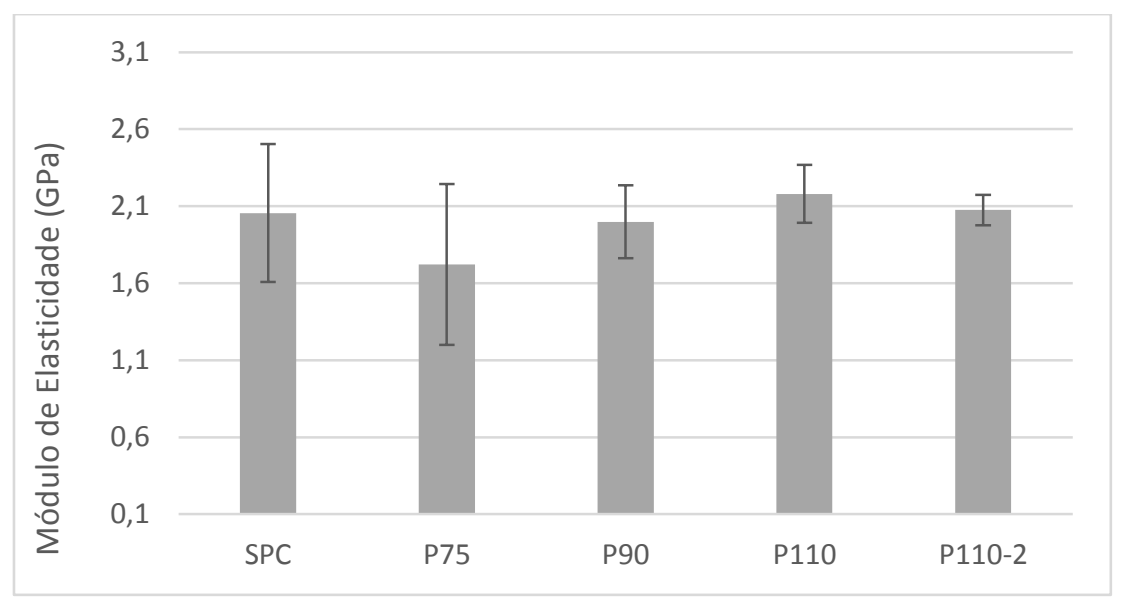

Figura 9: Módulo de elasticidade calculado via ensaio de viga livre para diferentes ciclos de pós cura.

Os valores calculados mostraram uma variação em torno de 1,72 a 2,18 GPa, sendo que de acordo com a literatura uma resina epóxi pode apresentar valores próximos a 3,00 GPa de módulo de elasticidade [18]. Entre as condições investigadas não foi observada nenhuma influência do ciclo de pós cura, pois todos os valores estão dentro da faixa de incerteza do próprio método de medição. Assim, estes resultados vão de acordo com as constatações mencionadas, onde dentro da faixa escolhida de pós cura não houve aumento considerável de ligações cruzadas que poderia refletir no módulo de elasticidade do material.

\section{CONCLUSÕES}

Os ciclos de pós cura utilizados neste artigo variaram entre $75^{\circ} \mathrm{C}$ a $110^{\circ} \mathrm{C}$. A primeira característica avaliada via DSC constatou que todas as Tgs $\infty$ estiverem na mesma faixa para o primeiro aquecimento. Ao analisar o segundo aquecimento no equipamento, o que submeteu as amostras inicialmente a outro ciclo de térmico de $200^{\circ} \mathrm{C}$, todas tiveram um aumento da $\mathrm{Tg} \infty$ na ordem de $35 \%$ do valor anterior. Assim, sugere-se que a temperaturas de pós cura mais elevadas do que as escolhidas neste trabalho o reflexo na $\mathrm{Tg} \infty$ da resina seria evidenciado.

Com a utilização da técnica de TGA foi constatada que a resina após curada tem uma forma de degradação térmica em etapas, onde $70 \%$ do material sofre degradação térmica na faixa de temperaturas de $330,9^{\circ} \mathrm{C}$ até $345,1^{\circ} \mathrm{C}$. Esta degradação foi atribuída a resina.

A amostra P90, a qual passou por um ciclo térmico de $90^{\circ} \mathrm{C}$ por um período de 1 hora após a cura, apresentou de acordo com as análises de TGA uma quantidade maior de agente endurecedor, proporcionando uma cura plena. De acordo com a cinética de cura nos estágios iniciais, as reações são controladas pela cinética de química dos reagentes e posteriormente o controle acontece por difusão. Quando a vitrificação ocorre, a reação torna-se a controlada por difusão, e a taxa de reação diminui em duas ou três ordens de grandeza abaixo daquelas nas regiões líquida ou borracha. Assim é sugerido que a quantidade de resina e agente endurecedor teve uma influência mais significativa na cura plena da amostra do que os ciclos de pós cura adotados neste trabalho. $\mathrm{O}$ excesso de agente de cura funcionou como um plastificante na matriz da resina, diminuindo os valores de dureza obtidos. Os ciclos de pós cura não alteraram a dureza da resina epóxi. Em termos de módulo de elasticidade, as resinas apresentaram valores entre 1,72 GPa e 2,18 GPa, e os ciclos de pós cura não tiverem nenhuma influência notável nesta propriedade.

\section{BIBLIOGRAFIA}

[1] SARI, M.G., SHAMSHIRI, M., RAMEZANZADEH, B., "Fabricating an epoxy composite coating with enhanced corrosion resistance through impregnation of functionalized graphene oxide-co-montmorillonite Nanoplatelet", Corrosion Science, v. 129, pp. 38-53, Dec. 2017.

[2] GARCIA, F.G., LEYVA, M.E., QUEIROZ, A.A.A., "Influência da Estrutura química do co-monômero nas propriedades mecânicas e adesivas de redes epoxídicas", Polímeros [online], v. 20, n.2, pp.148-152, Jun 2011.

[3] LEE, M.-C., HO, T.-H., WANG, C.-S., "Synthesis of tetrafunctional epoxy resins and their modification 
with polydimethylsiloxane for electronic application", Journal of Applied Polymer Science, v. 62: pp. 217225, Oct. 1996.

[4] GUADAGNO L., RAIMONDO M., VITTORIA V., et al., "Development of epoxy mixtures for application in aeronautics and aerospace", RSC Advances, v. 4, pp. 15474-15488, Mar. 2014.

[5] JIN L., LI X., PARK S.J., "Synthesis and application of epoxy resins: a review", Journal of Industrial and Engineering Chemistry, v. 29, pp. 1-11, Apr. 2015.

[6] MENEZES, G.W., MONTEIRO, S. N., ALMEIDA,D., et al., "Análise térmica da resina epóxi DGEBA/TETA para formulações diferentes da razão estequiométrica", Revista ABM, v. 1, n.1, pp. 12-16, Jul. 2016.

[7] PARDINI, L.C., LEVY NETO, F.. Compósitos estruturais: ciência e tecnologia, 1. ed., São Paulo, Edgar Blücher, 2006

[8] MENCZEL J.D., PRIME R.B., Thermal analysis of polymers, fundamentals and applications, 1 ed., New York, Wiley, 2009.

[9] AMERICAN SOCIETY FOR TESTING AND MATERIALS, "E793 - 06 Standard Test Method for Enthalpies of Fusion and Crystallization by Differential Scanning Calorimetry, crystallization, differential scanning calorimeter, DSC, energy, enthalpy, fusion, heat, melting” In: Annual Book of ASTM Standards, West Conshohocken, ASTM International, 2012.

[10] AMERICAN SOCIETY FOR TESTING AND MATERIALS, "E 1876-01 Standard test method fod dynamic youngs modulus, shear modulus, and poissons ratio by impulse excitation of vibration" In: Annual Book of ASTM Standards, West Conshohocken, ASTM International, 2001.

[11] FIORENTIN, T. A., Estudo de geração de ruído em reatores de núcleo de ar. Dissertação (mestrado), Universidade Federal de Santa Catarina, Florianópolis, SC, Brasil, 2007.

[12] PASCAULT, J.-P., SAUTEREAU, H., VERDU, J., et al., Thermosetting polymers., Marcel Dekker Inc., New York, Basel , 2002.

[13] CARBAS, R.J.C., SILVA, L.F.M.D., MARQUES, E.A.S., et al., "Effect of post-cure on the glass transition temperature and mechanical properties of epoxy adhesives", Journal of Adhesion Science and Technology, vol. 27, pp. 2542-2557, Oct. 2013.

[14] ZHANG, J., XU, Y.C., HUANG, P., "Effect of Cure Cycle on Curing Process and Hardness for Epoxy Resin”, Express Polymer Letters, v. 3, pp. 534-541, Jun. 2009.

[15] PLEPYS A.R., FARRIS R.J., "Evolution of residual stresses in three-dimensionally constrained epoxy resins", Polymer, v. 31, n. 10, pp.1932-1936, Oct. 1990.

[16] SHOKRIEH, M.M., AKBARI R., S., "Effect of Post-Cure Time on Residual Stress Distribution in Carbon/Epoxy Laminated Composites", The International Journal of Advanced Manufacturing Technology., vol. 5, pp. 13-18, Sept. 2012.

[17] AMARAL T.P., BARRA G.M.O., BARCIA F.O L., SOARES B.G., "Estudo das Propriedades de Compósitos de Polianilina e Resina Epoxídica", Polímeros, v. 11, n. 3, pp. 149-157, Oct. 2001.

[18] HULL, D., CLYNE, T. W, An introduction to composite materials, 2 ed. Cambridge Univ. Press, Cambridge, UK. 1996.

\section{ORCID}

Gabriel Benedet Dutra

Robson Tureck Zargiski

Thiago Antonio Fiorentin

Robin Bahr Junior

Pedro Zanetti Ribeiro Ferreira https://orcid.org/0000-0002-1095-7004

https://orcid.org/0000-0003-0436-5202

https://orcid.org/0000-0002-2559-7574

https://orcid.org/0000-0002-7396-0550

https://orcid.org/0000-0003-2484-0218 